\title{
A new formulation for the linearized Navier-Stokes equation
}

\author{
Y. Ouali*, A. Younes \\ Faculty of Science of Tunis, Tunis El Manar University - 2092, TUNISIA \\ *Corresponding author E-mail: ouali_yousra@yahoo.fr
}

Copyright (C)2014 Y. Ouali and A. Younes. This is an open access article distributed under the Creative Commons Attribution License, which permits unrestricted use, distribution, and reproduction in any medium, provided the original work is properly cited.

\begin{abstract}
This paper is devoted to study the Navier-Stokes equations by applying the curl and using a current function, we obtain a non-linear biharmonic problem where the pressure disappears and instead of the velocity, we are working with a scalar function. After a linearization, we obtain a sequence of linear problems. We study the existence and uniqueness of its solutions. Finally we show the convergence of the sequence of the linearized problems obtained to the non-linear one.
\end{abstract}

Keywords: Bi-Laplacian, Existence and uniqueness, Navier-Stokes equations.

\section{Introduction}

We consider the Navier-Stokes problem:

$(P)\left\{\begin{array}{rlrl}-\nu \Delta u+(u \nabla) u+\nabla P & =f & \text { in } & \Omega, \\ \operatorname{div} u=0 & \text { in } \quad \Omega, \\ u=0 & \text { on } \quad \Gamma,\end{array}\right.$

where $\Omega$ is a bounded and connected domain in $\mathbb{R}^{2}$ with lipschitz boundary $\Gamma=\partial \Omega$,

$\boldsymbol{u}$ the velocity and $\boldsymbol{p}$ the pressure.

$\nu$ is a positive parameter called kinematic viscosity and the corresponding function $f$ forces applied to the fluid is given.

After the application of the curl and using a current function, we obtain a non-linear biharmonic problem.

The variational formulation of the Navier-Stokes equations in the classic form is well studied in [2], [4], and [6]. A discretisation by Finite Element Methods of the problem is proposed by [4].

The standard discretization of the Stokes and Navier-Stokes equations in vorticity and stream function formulation by affine finite elements is known for its bad convergence. Amara.M and Bernardi.C in [1] present a modified discretization, they prove that the convergence is improved and they establish a priori error estimates.

The outline of the paper is as follows: 
In the second Section, we are concerned with the bi-harmonic equation by applying the curl and using a current function.

In Section 3, we study the sequence of linearized problems. We show the existence and uniqueness of their solutions .

In Section 4, we show the convergence of the sequence of solutions of the linearized problems obtained to the non-linear one.

In section 5 , we demonstrated the linear convergence.

\section{Application of rotational}

We have div $u=0$, then it can be written in the form $u=\operatorname{curl} \phi$ where $\phi$ is a scalar function called fairly regular stream function.

$$
\left\{\begin{array}{l}
u_{1}=\frac{\partial \phi}{\partial y} \\
u_{2}=-\frac{\partial \phi}{\partial x}
\end{array}\right.
$$

Then by applying the curl to our problem $(P)$, we will have:

curl $\Delta u=-\Delta^{2} \phi$,

$\operatorname{curl}(\nabla P)=0$,

$\operatorname{curl}((u . \nabla) u)=\frac{\partial}{\partial x}\left(u_{1} \frac{\partial u_{2}}{\partial x}+u_{2} \frac{\partial u_{2}}{\partial y}\right)-\frac{\partial}{\partial y}\left(u_{1} \frac{\partial u_{1}}{\partial x}+u_{2} \frac{\partial u_{1}}{\partial y}\right)$,

since

$\operatorname{div} u=0 \quad$ this implies that $\quad \frac{\partial u_{1}}{\partial x}=-\frac{\partial u_{2}}{\partial y}$,

and then, we obtain:

$\operatorname{curl}((u . \nabla) u)=-\frac{\partial \phi}{\partial y} \frac{\partial \Delta \phi}{\partial x}+\frac{\partial \phi}{\partial x} \frac{\partial \Delta \phi}{\partial y}$.

The equation becomes

$\nu \Delta^{2} \phi-\frac{\partial \phi}{\partial y} \frac{\partial \Delta \phi}{\partial x}+\frac{\partial \phi}{\partial x} \frac{\partial \Delta \phi}{\partial y}=\operatorname{curl} f$

and we have the following problem

$(Q)\left\{\begin{aligned} \nu \Delta^{2} \phi-\frac{\partial \phi}{\partial y} \frac{\partial \Delta \phi}{\partial x}+\frac{\partial \phi}{\partial x} \frac{\partial \Delta \phi}{\partial y} & =\operatorname{curl} f \quad \text { in } \quad \Omega, \\ \phi & =0 \quad \text { on } \quad \Gamma, \\ \frac{\partial \phi}{\partial x}=\frac{\partial \phi}{\partial y} & =0 \quad \text { on } \quad \Gamma .\end{aligned}\right.$

A linearization gives:

$\nu \Delta^{2} \phi_{n+1}-\frac{\partial \phi_{n}}{\partial y} \frac{\partial \Delta \phi_{n+1}}{\partial x}+\frac{\partial \phi_{n}}{\partial x} \frac{\partial \Delta \phi_{n+1}}{\partial y}=\operatorname{curl} f$.

We set

$$
c_{n}=\frac{\partial \phi_{n}}{\partial y}, d_{n}=\frac{\partial \phi_{n}}{\partial x},
$$


we have:

$\nu \Delta^{2} \phi_{n+1}-c_{n} \frac{\partial \Delta \phi_{n+1}}{\partial x}+d_{n} \frac{\partial \Delta \phi_{n+1}}{\partial y}=\operatorname{curl} f$.

Therefore, our problem is:

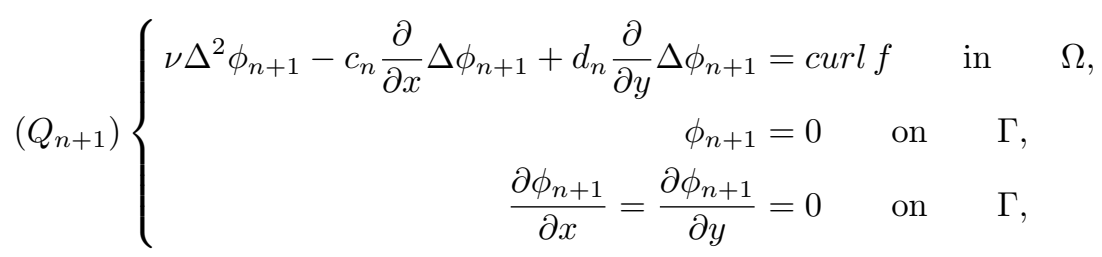

\section{Variational formulation}

We multiply both sides of the first equation of $\left(Q_{n+1}\right)$ by a test function $v \in V=H_{0}^{2}(\Omega)$ and integrating over $\Omega$, we have the following variational problem:

$(Q V)_{n+1}\left\{\begin{array}{l}\text { Find } \phi_{n+1} \in V \text { such as } \\ a\left(\phi_{n+1}, v\right)=L(v), \quad \forall v \in V,\end{array}\right.$

where $a(.,$.$) is a bilinear form on V \times V$ given by:

$a(u, v)=a_{0}(u, v)+a_{n}(u, v)$,

where

$a_{0}(u, v)=\nu \int_{\Omega} \Delta u \Delta v d X$,

$a_{n}(u, v)=\int_{\Omega} \Delta u\left(\frac{\partial \phi_{n}}{\partial y} \frac{\partial v}{\partial x}-\frac{\partial \phi_{n}}{\partial x} \frac{\partial v}{\partial y}\right) d X$,

and $L($.$) is a linear form on V$ defined by the following expression:

$L(v)=\int_{\Omega} \operatorname{curl} f v d X$.

For the existence and uniqueness of the solution, we need this lemma:

Lemma 3.1. [5], $\forall u \in H^{2}(\Omega) \cap H_{0}^{1}(\Omega)$, there exist $c^{\prime}(\Omega)>0$ such that

$\|u\|_{H^{2}}^{2} \leq c^{\prime}(\Omega)\|\Delta u\|_{2}^{2} \leq c^{*}\|\Delta u\|_{2}^{2}$.

where $c^{*}$ will be chosen later.

Theorem 3.2. For $f \in H^{1}(\Omega)$ and curl $f$ small enough: $\|$ curl $f \|_{2}<c(\nu)$, the problem $(Q V)_{n+1}$ has a unique solution $\phi_{n+1} \in V$.

Proof. 1. Continuity of $a$ :

We show the continuity and coercivity of the bilinear form $a(.,$.$) .$

We put $u=\phi_{n+1}$ and we deal with each term separately, we begin by:

$$
\begin{aligned}
\left|a_{0}(u, v)\right| & \leq \nu\|\Delta u\|_{2}\|\Delta v\|_{2}, \\
& \leq \nu\|u\|_{H^{2}}\|v\|_{H^{2}} .
\end{aligned}
$$

And using the continuous injection of $H^{1}(\Omega)$ in $L^{4}(\Omega), a_{n}(.,$.$) will be bounded as bellow$

$$
\begin{aligned}
\left|a_{n}(u, v)\right| & =\left|\int_{\Omega} \Delta u\left(\nabla v \wedge \nabla \phi_{n}\right) d X\right|, \\
& \leq\|\Delta u\|_{2}\|\nabla v\|_{L^{4}}\left\|\nabla \phi_{n}\right\|_{L^{4}}, \\
& \leq c\|\Delta u\|_{2}\|\nabla v\|_{H^{1}}\left\|\nabla \phi_{n}\right\|_{H^{1}}, \\
& \leq c\left\|\phi_{n}\right\|_{H^{2}}\|u\|_{H^{2}}\|v\|_{H^{2}} .
\end{aligned}
$$


Therefore,

$|a(u, v)| \leq \mathcal{C}_{n}\|u\|_{H^{2}}\|v\|_{H^{2}}$

where $\mathcal{C}_{n}$ is a constant which depends on $n$ given by:

$$
\mathcal{C}_{n}=\nu+c\left\|\phi_{n}\right\|_{H^{2}}
$$

This implies that, for each fixed $n, a(.,$.$) is continuous on V$.

\section{Coercivity of $a$ :}

We have:

$a_{0}(u, u)=\nu\|\Delta u\|_{2}^{2}$,

and taking $v=u$ in (7), we obtain:

$\left|a_{n}(u, u)\right| \leq c\left\|\phi_{n}\right\|_{H^{2}}\|u\|_{H^{2}}^{2}$,

then using (5)

$$
\begin{aligned}
a(u, u) & \geq \nu\|\Delta u\|_{2}^{2}-c\|u\|_{H^{2}}^{2}\left\|\phi_{n}\right\|_{H^{2}}, \\
& \geq \frac{\nu}{c^{\prime}(\Omega)}\|u\|_{H^{2}}^{2}-c\|u\|_{H^{2}}^{2}\left\|\phi_{n}\right\|_{H^{2}}, \\
& \geq\left(\frac{\nu}{c^{\prime}(\Omega)}-c\left\|\phi_{n}\right\|_{H^{2}}\right)\|u\|_{H^{2}}^{2} .
\end{aligned}
$$

To get the coercivity, we should have:

$\frac{\nu}{c^{\prime}(\Omega)}-c\left\|\phi_{n}\right\|_{H^{2}}>0, \quad \forall n \in \mathbb{N}$,

which means that

$\left\|\phi_{n}\right\|_{H^{2}}<\frac{\nu}{c^{\prime}(\Omega) c}, \quad \forall n \in \mathbb{N}$.

Let

$\alpha^{*}=\frac{\nu}{2 c^{\prime}(\Omega) c}$.

We take $\phi_{0} \in B_{\alpha^{*}}$ where $B_{\alpha}=\left\{v \in H_{0}^{2}(\Omega) ;\|v\|_{H^{2}} \leq \alpha\right\}$ and assume that $\phi_{n} \in B_{\alpha^{*}}$, we must show by induction that: $\phi_{n+1} \in B_{\alpha^{*}}$.

Indeed, we have, if we put $u=\phi_{n+1}$ :

$a(u, u)=L(u)=\int_{\Omega} \operatorname{curl} f u d X$,

$a(u, u) \leq\|\operatorname{curl} f\|_{2}\|u\|_{2}$,

then

$\left(\frac{\nu}{c^{\prime}(\Omega)}-c\left\|\phi_{n}\right\|_{H^{2}}\right)\|u\|_{H^{2}}^{2} \leq\|\operatorname{curl} f\|_{2}\|u\|_{H^{2}}$,

and

$\left(\frac{\nu}{c^{\prime}(\Omega)}-c\left\|\phi_{n}\right\|_{H^{2}}\right)\|u\|_{H^{2}} \leq\|\operatorname{curl} f\|_{L^{2}}$, 
$\|u\|_{H^{2}} \leq \frac{\| \text { curl } f \|_{2}}{\frac{\nu}{c^{\prime}(\Omega)}-c\left\|\phi_{n}\right\|_{H^{2}}}$.

If we assume that:

$\|$ curl $f \|_{2}<\frac{\nu^{2}}{4 c^{\prime}(\Omega)^{2} c}$,

then we have :

$\|u\|_{H^{2}} \leq \frac{\|\operatorname{curl} f\|_{2}}{\frac{\nu}{c^{\prime}(\Omega)}-c \alpha^{*}} \leq \alpha^{*}$,

which implies that $a(.,$.$) is coercive on V$.

\section{Continuity of $L$ :}

In the other hand, the linear form $L$ is continuous:

$\|L(v)\|_{2} \leq\|\operatorname{curl} f\|_{2}\|v\|_{2}$.

Then, using Lax-Milgram Theorem, the problem $(Q V)_{n+1}$ has a unique solution $\phi_{n+1} \in V$.

\section{Convergence of the sequence}

The sequence $\left(\phi_{n}\right)_{n \in \mathbb{N}}$ obtained in the preceding section verifies:

$\left\|\phi_{n}\right\|_{V} \leq \alpha^{*}, \quad \forall n \geq 0$

which implies that the sequence $\left(\phi_{n}\right)_{n \in \mathbb{N}}$ is bounded in $V$.

Then there exist a subsequence that converges weakly to $w$ in $V$.

Since the injection of $V$ in $H_{0}^{1}(\Omega)$ is continuous, there exists a subsequence still noted $\phi_{n}$ which converges strongly to $w$ in $H_{0}^{1}(\Omega)$.

For the convergence, we need this regularity result:

Lemma 4.1. Assume that $\Omega$ is of class $\mathcal{C}^{2}$ and $\phi_{0} \in H_{0}^{2}(\Omega) \cap H^{3}(\Omega)$, we have:

$$
\forall n \in \mathbb{N}, \quad \phi_{n+1} \in H_{0}^{2}(\Omega) \cap H^{3}(\Omega) .
$$

Proof. The problem $\left(Q_{n+1}\right)$ can be written as

$$
\left\{\begin{array}{l}
-\nu \Delta \omega_{n+1}+c_{n} \frac{\partial \omega_{n+1}}{\partial x}-d_{n} \frac{\partial \omega_{n+1}}{\partial y}=-\operatorname{curl} f \quad \text { in } \Omega, \\
\Delta \phi_{n+1}=\omega_{n+1} \quad \text { in } \Omega, \quad(2) \\
\phi_{n+1} \in H_{0}^{2}(\Omega) .
\end{array}\right.
$$

The variational formulation of (1) is

$\left\{\begin{array}{l}\text { Find } \omega_{n+1} \in H_{0}^{1}(\Omega) \text { such as } \\ A_{n}\left(\omega_{n+1}, v\right)=l(v), \quad \forall v \in H_{0}^{1}(\Omega),\end{array}\right.$

where

$A_{n}\left(\omega_{n+1}, v\right)=\nu \int_{\Omega} \nabla \omega_{n+1} \nabla v d X+\int_{\Omega} \omega_{n+1}\left(d_{n} \frac{\partial v}{\partial y}-c_{n} \frac{\partial v}{\partial x}\right) d X$,

$l(v)=-\int_{\Omega} \operatorname{curl} f v d X$. 
For the coercivity of $A_{n}$, we have:

$$
\begin{aligned}
A_{n}(v, v) & =\nu \int_{\Omega}|\nabla v|^{2} d X+\int_{\Omega} v\left(\nabla v \wedge \nabla \omega_{n}\right) d X \\
& \geq \nu\|\nabla v\|_{2}^{2}-c\|\nabla v\|_{2}\|v\|_{H^{1}}\left\|\nabla \omega_{n}\right\|_{H^{1}} \\
& \geq \nu c_{1}\|v\|_{H^{1}}^{2}-c \alpha^{*}\|v\|_{H^{1}}^{2} .
\end{aligned}
$$

It suffices to choose in inequality (5): $c^{*}>\frac{1}{2 c_{1}}$.

And by Lax-Milgram Theorem, we have $\omega_{n+1} \in H^{1}(\Omega)$.

The theory of regularity for weak solutions of the laplace problem applied to the variational formulation of (2) gives $\phi_{n+1} \in H^{3}(\Omega)$.

Then, we have this result of convergence :

Lemma 4.2. 1. We have, $\forall v \in H_{0}^{2}(\Omega) \cap H^{3}(\Omega)$ :

$$
\lim _{n \rightarrow+\infty} a_{0}\left(\phi_{n+1}, v\right)=a_{0}(w, v)
$$

2. We have, $\forall v \in H_{0}^{2}(\Omega) \cap H^{3}(\Omega)$ :

$$
\lim _{n \rightarrow+\infty} a_{n}\left(\phi_{n+1}, v\right)=a_{\infty}(w, v)=\int_{\Omega} \Delta w(\nabla v \wedge \nabla w) d X .
$$

Proof. 1. We have:

$$
\begin{aligned}
\left|a_{0}\left(\phi_{n+1}, v\right)-a_{0}(w, v)\right| & =\left|\nu \int_{\Omega} \Delta\left(\phi_{n+1}-w\right) \Delta v d X\right| \\
& \leq \nu\left\|\nabla\left(\phi_{n+1}-w\right)\right\|_{2}\|v\|_{H^{3}},
\end{aligned}
$$

then, we obtain the result.

2. On the other hand, we have:

$\left|a_{n}\left(\phi_{n+1}, v\right)-a_{\infty}(w, v)\right|=T_{1_{n}}+T_{2_{n}}$

where

$T_{1_{n}}=\left|\int_{\Omega}\left(\Delta \phi_{n+1}-\Delta w\right)\left(\nabla v \wedge \nabla \phi_{n}\right) d X\right|$,

and

$T_{2_{n}}=\left|\int_{\Omega} \Delta w \cdot\left[\nabla v \wedge\left(\nabla \phi_{n}-\nabla w\right)\right] d X\right|$

By Green formula we have :

$T_{1_{n}}=\left|\int_{\Omega}\left(\nabla \phi_{n+1}-\nabla w\right) \nabla\left(\nabla v \wedge \nabla \phi_{n}\right) d X\right|$

which gives :

$T_{1_{n}} \leq \sum_{i, j=1}^{2}\left|\int_{\Omega} \frac{\partial\left(\phi_{n+1}-\omega\right)}{\partial x_{i}} \frac{\partial^{2} v}{\partial x_{i} \partial x_{j}} \frac{\partial\left(\phi_{n}\right)}{\partial x_{j}} d X\right|+\sum_{i, j=1}^{2}\left|\int_{\Omega} \frac{\partial\left(\phi_{n+1}-\omega\right)}{\partial x_{i}} \frac{\partial v}{\partial x_{j}} \frac{\partial^{2}\left(\phi_{n}\right)}{\partial x_{i} \partial x_{j}} d X\right|$.

and

$T_{2_{n}} \leq \sum_{i, j=1}^{2}\left|\int_{\Omega} \frac{\partial\left(\phi_{n}-\omega\right)}{\partial x_{i}} \frac{\partial^{2} \omega}{\partial x_{i} \partial x_{j}} \frac{\partial v}{\partial x_{j}} d X\right|$ 
According to the Sobolev imbedding Theorem the space $H^{1}(\Omega)$ is continuously imbedded in $L^{4}(\Omega)$ for $n=2$.

Then by the Hölder's inequality we have for $\omega, v, \phi_{n} \in H_{0}^{2}(\Omega) \cap H^{3}(\Omega)$ :

$\frac{\partial \omega}{\partial x_{i}} \frac{\partial^{2} v}{\partial x_{i} \partial x_{j}} \frac{\partial\left(\phi_{n+1}-\omega\right)}{\partial x_{j}} \in L^{1}(\Omega), \quad 1 \leq i, j \leq 2$,

with for $T_{1 n}$

$$
\begin{aligned}
\left|\int_{\Omega} \frac{\partial \phi_{n}}{\partial x_{i}} \frac{\partial^{2} v}{\partial x_{i} \partial x_{j}} \frac{\partial\left(\phi_{n+1}-\omega\right)}{\partial x_{j}} d X\right| & \leq\left\|\frac{\partial^{2} v}{\partial x_{i} \partial x_{j}}\right\|_{4}\left\|\frac{\partial \phi_{n}}{\partial x_{i}}\right\|_{4}\left\|\frac{\partial\left(\phi_{n+1}-\omega\right)}{\partial x_{j}}\right\|_{2} \\
& \leq \mathcal{C}\left\|\frac{\partial^{2} v}{\partial x_{i} \partial x_{j}}\right\|_{H^{1}}\left\|\frac{\partial \phi_{n}}{\partial x_{i}}\right\|_{H^{1}}\left\|\frac{\partial\left(\phi_{n+1}-\omega\right)}{\partial x_{j}}\right\|_{2} \\
& \leq \mathcal{C}\|v\|_{H^{2}}\left\|\phi_{n}\right\|_{H^{2}}\left\|\frac{\partial\left(\phi_{n}-\omega\right)}{\partial x_{j}}\right\|_{2},
\end{aligned}
$$

and

$$
\begin{aligned}
\left|\int_{\Omega} \frac{\partial^{2} \phi_{n}}{\partial x_{i} \partial x_{j}} \frac{\partial v}{\partial x_{j}} \frac{\partial\left(\phi_{n+1}-\omega\right)}{\partial x_{i}} d X\right| & \leq\left\|\frac{\partial^{2} \phi_{n}}{\partial x_{i} \partial x_{j}}\right\|_{4}\left\|\frac{\partial v}{\partial x_{j}}\right\|_{4}\left\|\frac{\partial\left(\phi_{n+1}-\omega\right)}{\partial x_{i}}\right\|_{2} \\
& \leq \mathcal{C}\left\|\frac{\partial^{2} \phi_{n}}{\partial x_{i} \partial x_{j}}\right\|_{H^{1}}\left\|\frac{\partial v}{\partial x_{j}}\right\|_{H^{1}}\left\|\frac{\partial\left(\phi_{n+1}-\omega\right)}{\partial x_{i}}\right\|_{2} \\
& \leq \mathcal{C}\|v\|_{H^{2}}\left\|\phi_{n}\right\|_{H^{2}}\left\|\frac{\partial\left(\phi_{n+1}-\omega\right)}{\partial x_{i}}\right\|_{2},
\end{aligned}
$$

and for $T_{2 n}$ we have

$$
\begin{aligned}
\left|\int_{\Omega} \frac{\partial^{2} \omega}{\partial x_{i} \partial x_{j}} \frac{\partial v}{\partial x_{j}} \frac{\partial\left(\phi_{n}-\omega\right)}{\partial x_{i}} d X\right| & \leq\left\|\frac{\partial^{2} \omega}{\partial x_{i} \partial x_{j}}\right\|_{4}\left\|\frac{\partial v}{\partial x_{j}}\right\|_{4}\left\|\frac{\partial\left(\phi_{n}-\omega\right)}{\partial x_{i}}\right\|_{2} \\
& \leq \mathcal{C}\left\|\frac{\partial^{2} \omega}{\partial x_{i} \partial x_{j}}\right\|_{H^{1}}\left\|\frac{\partial v}{\partial x_{j}}\right\|_{H^{1}}\left\|\frac{\partial\left(\phi_{n}-\omega\right)}{\partial x_{i}}\right\|_{2} \\
& \leq \mathcal{C}\|v\|_{H^{2}}\|\omega\|_{H^{2}}\left\|\frac{\partial\left(\phi_{n}-\omega\right)}{\partial x_{i}}\right\|_{2},
\end{aligned}
$$

Then, by the strongly convergence in $H_{0}^{1}(\Omega)$, we will have

$$
\lim _{n \rightarrow+\infty} a_{n}\left(\phi_{n+1}, v\right)=a_{\infty}(w, v) .
$$

\section{Proposition 1. $w$ is a solution of $Q$.}

Proof. It follows from Lemma (4.1) that:

$\lim _{n \rightarrow+\infty} a_{0}\left(\phi_{n+1}, v\right)+a_{n}\left(\phi_{n+1}, v\right)=a_{0}(w, v)+a_{\infty}(w, v)=L(v)$.

Which gives:

$\nu \int_{\Omega} \Delta w \Delta v d X+\int_{\Omega} \Delta w(\nabla v \wedge \nabla w) d X=\int_{\Omega} \operatorname{curlf} v d X$,

then

$\int_{\Omega}\left(\nu \Delta^{2} w-\frac{\partial w}{\partial y} \frac{\partial \Delta w}{\partial x}+\frac{\partial w}{\partial x} \frac{\partial \Delta w}{\partial y}-\operatorname{curl} f\right) v d X=0, \quad \forall v \in H_{0}^{2}(\Omega)$,

then

$\nu \Delta^{2} w-\frac{\partial w}{\partial y} \frac{\partial \Delta w}{\partial x}+\frac{\partial w}{\partial x} \frac{\partial \Delta w}{\partial y}=\operatorname{curl} f, \quad \forall w \in H_{0}^{2}(\Omega)$.

So we can conclude that $w$ is the solution of $Q$. 


\section{Linear convergence}

For this part, we set

$$
w_{n+1}=\phi_{n+1}-w,
$$

we have the following result:

Proposition 2. For $w_{n+1} \in H^{2}(\Omega)$, there exist a constant $C$ such that:

$\left\|w_{n+1}\right\|_{H^{2}} \leq C\left\|w_{n}\right\|_{H^{2}}$.

Proof. Taking the difference between the problem $\left(Q_{n+1}\right)$ and the problem $(Q)$, we have:

$\nu \Delta^{2} w_{n+1}-\frac{\partial \phi_{n}}{\partial y} \frac{\partial\left(\Delta w_{n+1}\right)}{\partial x}+\frac{\partial \phi_{n}}{\partial x} \frac{\partial\left(\Delta w_{n+1}\right)}{\partial y}=F_{n}$,

where

$F_{n}=\frac{\partial(\Delta w)}{\partial x} \frac{\partial w_{n}}{\partial y}-\frac{\partial(\Delta w)}{\partial y} \frac{\partial\left(w_{n}\right)}{\partial x}$.

The variational formulation gives us:

$$
\begin{aligned}
\beta\left\|w_{n+1}\right\|_{H^{2}}^{2} \leq a\left(w_{n+1}, w_{n+1}\right) & \leq \int_{\Omega}\left|F_{n}\right| w_{n+1} d X, \\
& =\int_{\Omega}\left|\Delta w\left(\nabla w_{n} \wedge \nabla w_{n+1}\right)\right| d X, \\
& \leq\|\Delta w\|_{2}\left\|\nabla w_{n}\right\|_{4}\left\|\nabla w_{n+1}\right\|_{4}, \\
& \leq c\|\Delta w\|_{2}\left\|\nabla w_{n}\right\|_{H^{1}}\left\|\nabla w_{n+1}\right\|_{H^{1}}, \\
& \leq c^{\prime}\left\|w_{n}\right\|_{H^{2}}\left\|w_{n+1}\right\|_{H^{2}},
\end{aligned}
$$

then, we obtain:

$\left\|w_{n+1}\right\|_{H^{2}} \leq C\left\|w_{n}\right\|_{H^{2}}$

where $C$ is given by:

$C=\frac{c^{\prime}}{\beta}$

and $\beta$ the constant of the coercivity.

Which implies the linear convergence.

\section{Conclusion}

In this paper, we studied the Navier-Stokes equations by applying the curl and using a current function through the application of rotational, we obtained a non-linear biharmonic problem.

After a linearization, we proved the existence and uniqueness of weak solution of the variational formulation using Lax-Milgram Theorem and which we can compute by finite element method.

And in a second part we showed the convergence of the sequence as well as the linear convergence.

\section{Acknowledgements}

Thanks to all that participated in this study. 


\section{References}

[1] Amara.M, Bernardi.C, "Convergence Of A Finite Element Discretization of The Navier-Stokes Equations In Vorticity And Stream Function Formulation", M2AN, Vol.33, No.5, (1999), pp.1033-1056.

[2] Amara.M, Capatina-Papaghiuc.D, Chacon-Vera.E, Trijullo.D, "Vorticity velocity pressure formulation for Navier-Stokes equations", Comput. Vis. Sci, No.6, (2004), pp.47-52.

[3] Bernardi.C, Mady.Y, Rapetti.F, "Discretisations variationnelles de problmes aux limites elliptiques", Springer-Verlag Berlin Heidelberg, (2004).

[4] Girault.V, Raviart.P-A, "Finite Element Methods for the Navier-Stokes Equations, Theory and Algorithms", SpringerVerlag, (1986).

[5] Ndlec.J-C, "Cours d'Analyse Numrique", Ecole Nationale de Ponts et Chausses.

[6] Temam.R, "Navier-Stokes Equations, Theory and Numerical Analysis", North-Holland, Amsterdam, (1984). 